\title{
Higher-order topological insulators, topological pumps and the quantum Hall effect in high dimensions
}

\author{
Ioannis Petrides and Oded Zilberberg \\ Institute for Theoretical Physics, ETH Zürich, 8093 Zürich, Switzerland
}

(Received 3 December 2019; accepted 14 May 2020; published 4 June 2020)

\begin{abstract}
Topological insulators are materials with spectral bands associated with an integer-valued index, manifesting through quantized bulk phenomena and robust boundary effects. In this Rapid Communication, we demonstrate that higher-order topological insulators are descendants from a high-dimensional chiral semimetal. Specifically, we apply dimensional reduction to an ancestor four-dimensional Chern insulator, and obtain two-dimensional (2D) second-order topological insulators when the former becomes chiral. Correspondingly, we derive the quantized charge accumulation at the corners of the $2 \mathrm{D}$ descendants and relate it to the topological index-the second Chern number - of the ancestor model. Our results provide a clear connection between the boundary states of higher-order topological insulators and topological pumps-the latter being dynamical realizations of the quantum Hall effect in high dimensions.
\end{abstract}

DOI: 10.1103/PhysRevResearch.2.022049

Over the past decades, the unique properties of topological insulators (TIs) led to many theoretical and experimental advances [1-3]. TIs have energy bands that are characterized by a nonlocal quantity, a topological index, which determines the bulk and boundary properties. The quantization of the topological index usually relies on local symmetries [4-6], symmorphic or nonsymmorphic crystalline symmetries [7,8], or even quasiperiodic order [9-12]. The resulting TIs are extensively studied and classified according to the presence or absence of such symmetries [4-6,13,14] using a plethora of methods, such as $K$ theory [4,15,16], nonlinear $\sigma$-model analysis [5,6,17], and dimensional reduction [2].

A paradigmatic example of a TI is the Chern insulator (CI), appearing in even dimensions. CIs exhibit quantized bulk transport responses $[18,19]$ proportional to the topological indices (Chern numbers) characterizing their spectra. Interestingly, using dimensional reduction, a CI in $d$ dimensions is mapped to a family of models in $d-m$ dimensions, dubbed the "descendant pump family". A periodic and adiabatic scan over the descendant pump family similarly results in quantized bulk transport that is proportional to the Chern numbers of the ancestor CI. Archetypical examples of such mapping are the [two-dimensional to one-dimensional $(2 \mathrm{D} \rightarrow 1 \mathrm{D})]$ reduction of a 2D CI to Thouless's 1D topological pump [20-24] and the [four-dimensional (4D) $\rightarrow$ 2D] reduction of a $4 \mathrm{D} \mathrm{CI}$ to $2 \mathrm{D}$ topological pumps $[18,25,26]$, see Table I.

The topological bulk responses of the ancestor CI have associated boundary phenomena where dispersive states of

Published by the American Physical Society under the terms of the Creative Commons Attribution 4.0 International license. Further distribution of this work must maintain attribution to the author(s) and the published article's title, journal citation, and DOI. co-dimension $h$ cross the gap, i.e., states localized in $h$ dimensions but extended in the remaining. Crucially, under dimensional reduction, the boundary states of the CI are mapped to the boundaries of the descendant pump family. For example, the Chern numbers of a 4D CI imply boundary states with codimensions $h=1$ and $h=2$ that, after dimensional reduction, are mapped to the edges and corners of the descendant $2 \mathrm{D}$ pump family [26].

Although CIs and topological pumps share equivalent Chern numbers, a relationship between topological indices in different classes and dimensions is obtained by imposing symmetry constraints when performing dimensional reduction. For example, the $[4 D \rightarrow$ three-dimensional (3D)] reduction of a $4 \mathrm{D}$ CI yields $3 \mathrm{D} \mathbb{Z}_{2}$ TIs when time-reversal (T.R.) symmetry is imposed [27], see Table I. This allows for the derivation of a $\mathbb{Z}_{2}$ index, characterizing the descendant 3D family, directly from the second Chern number of the ancestor $4 \mathrm{D}$ model. Correspondingly, the surface states of the $3 \mathrm{D} \mathbb{Z}_{2}$ TI descend from the $h=1$ boundary states of the 4D ancestor model, i.e., the boundary states related to the second Chern number.

Recently, zero-dimensional (0D) boundary states, i.e., states of co-dimension $h=2$, carrying a quantized charge $\pm 1 / 2$ were predicted in $2 \mathrm{D}$ materials [26,28-41]. This led to a new class of TIs, dubbed "higher-order TIs" where a $d$-dimensional insulator has nontrivial boundary phenomena manifesting at its $d-h$ boundary with $h>1$. The appearance of such states was explained using the modern theory of polarization, which was extended to higher multipole moments, and charge quantization was imposed by the underlying symmetries of the system [32].

In this Rapid Communication, we reveal a connection among higher-order TIs, descendant pump families, and highdimensional CIs. Specifically, we show that 2D second-order TIs are the 2D descendants of a 4D chiral semimetal. We do so by first defining an ancestor 4D CI with well-defined first 
TABLE I. Mapping between models in different dimensions and symmetry classes.

\begin{tabular}{|c|c|c|}
\hline Reduction & Ancestor Model & Descendant family \\
\hline $2 \mathrm{D} \rightarrow 1 \mathrm{D}$ & chiral $\left(\begin{array}{l}2 \mathrm{D} \text { Chern Insulator } \\
2 \mathrm{D} \text { Chiral Semimetal }\end{array}\right.$ & $\begin{array}{l}1 \mathrm{D} \text { pump } \\
1 \mathrm{D} \mathbb{Z} \text {-insulator }\end{array}$ \\
\hline $4 \mathrm{D} \rightarrow 3 \mathrm{D}$ & T.R. $\left(\begin{array}{l}\text { 4D Chern Insulator } \\
4 \mathrm{D} \text { T.R. Insulator }\end{array}\right.$ & $\begin{array}{l}\text { 3D pump } \\
\text { 3D } \mathbb{Z}_{2} \text {-insulator }\end{array}$ \\
\hline $4 \mathrm{D} \rightarrow 2 \mathrm{D}$ & chiral $\left(\begin{array}{l}4 \mathrm{D} \text { Chern Insulator } \\
4 \mathrm{D} \text { Chiral Semimetal }\end{array}\right.$ & $\begin{array}{l}2 \mathrm{D} \text { pump } \\
2 \mathrm{D} \text { second-order TI }\end{array}$ \\
\hline
\end{tabular}

and second Chern numbers and then applying $(4 \mathrm{D} \rightarrow 2 \mathrm{D})$ reduction to obtain the descendant 2D pump family [18,25,26]. We, then, show that the topological index, associated bulk responses, and the corresponding boundary phenomena of the 4D CI directly imply the properties of second-order TIs in the limit where the former becomes chiral. Respectively, we show that the descendant 2D family is divided into regions in parameter space separated by (bulk- or edge-) gap closures; these regions are distinguished by the appearance of midgap OD states, localized at the corners. We derive the quantization of corner charge by extending the formula of Jackiw-Rebbi [42] to the 2D descendants and connecting it to the second Chern flux of the ancestor 4D Hamiltonian. Finally, using dimensional reduction, we generate various higher-order TIs solely via flux insertions through different planes of the ancestor model, leading to a simple procedure for finding new models for material realizations.

We consider a tight-binding model describing spinless charged particles moving on a 4D hypercubic lattice in the presence of a magnetic field [see Fig. 1(a)],

$$
\hat{H}^{4 \mathrm{D}}=\sum_{\mathbf{m}}\left[\hat{H}_{x z}(\mathbf{m})+\hat{H}_{y w}(\mathbf{m})+\Delta \hat{H}_{x y}^{b}(\mathbf{m})\right],
$$

where $\mathbf{m}=\left(m_{x}, m_{y}, m_{z}, m_{w}\right)$ is a $4 \mathrm{D}$ lattice vector and $\hat{H}_{\mu \nu}(\mathbf{m})$ describes a 2D Creutz lattice $[10,43,44]$ on the $\mu \nu$ plane [cf. Figs. 1(a) and 1(b)]. In the Landau gauge, each Creutz model is written as $\hat{H}_{\mu \nu}(\mathbf{m})=\hat{T}_{\mu \nu}+\hat{T}_{\mu \nu}^{\dagger}$ with

$$
\begin{aligned}
\hat{T}_{\mu \nu}= & t_{\mu \nu}\left(e^{-i \pi m_{\mu}} c_{\mathbf{m}+\mathbf{e}_{\mu}+\mathbf{e}_{v}}^{\dagger} c_{\mathbf{m}}+e^{i \pi m_{\mu}} c_{\mathbf{m}+\mathbf{e}_{\mu}-\mathbf{e}_{\nu}}^{\dagger} c_{\mathbf{m}}\right) \\
& +t_{\mu} c_{\mathbf{m}+\mathbf{e}_{\mu}}^{\dagger} c_{\mathbf{m}},
\end{aligned}
$$

where $\mathbf{e}_{\mu}$ is a lattice unit vector in direction $\mu, t_{\mu}$ is the nearest-neighbor hopping amplitude, $t_{\mu \nu}$ is the next-nearestneighbor hopping amplitude, and the magnetic flux is incorporated using Peierls' substitution [45]. The third term in Eq. (1) denotes the threading of $b$ flux through each $x y$ plaquette,

$$
\Delta \hat{H}_{x y}^{b}(\mathbf{m})=\left(e^{i m_{x} b}-1\right) \hat{T}_{y w}+\text { H.c. }
$$

Finally, a potential $\hat{V}(\mathbf{m})=(-1)^{m_{x}+m_{y}} V_{0} c_{\mathbf{m}}^{\dagger} c_{\mathbf{m}}$ with $V_{0}$ as a constant (similarly when $t_{z} \neq 0 \neq t_{w}$ ) gaps the spectrum, and the 4D model is a CI with well-defined first and second Chern numbers.

The chosen gauge in Eq. (2) leaves the Hamiltonian $\hat{H}^{4 \mathrm{D}}$ invariant under lattice translations in the $z$ and $w$ directions. We can, therefore, write Eq. (1) in terms of the quasimomenta (a)

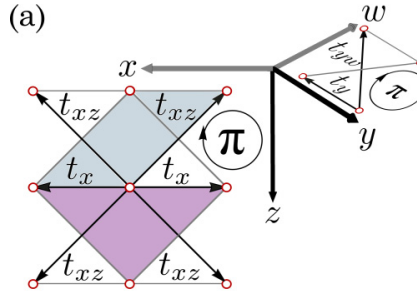

(b)

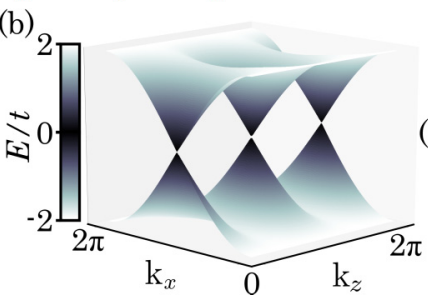

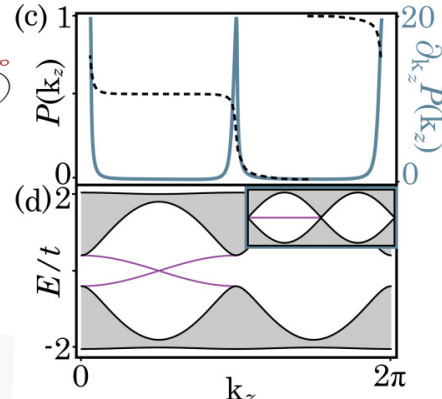

(e)

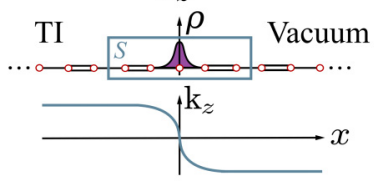

FIG. 1. Ancestor model and $(2 \mathrm{D} \rightarrow 1 \mathrm{D})$ reduction. (a) The $4 \mathrm{D}$ model [cf. Eq. (1)] made out of 2D Creutz lattices. Each triangular (and parallelogram) plaquette is threaded with a $\pi$ flux [cf. Eq. (2)]. (b) The energy spectrum of $\hat{H}_{x z}$ [cf. Eq. (2)], showing two Dirac cones. (c) The bulk dipole $P_{x}\left(k_{z}\right)$ (dashed line), associated with $\hat{h}_{x}\left(k_{z}\right)$ and its derivative $\partial_{\mathrm{k}_{z}} P_{x}\left(k_{z}\right)$ (solid line). The total area under $\partial_{\mathrm{k}_{z}} P_{x}\left(k_{z}\right)$ is equal to the first Chern number $c_{1}$ of the ancestor model. (d) The open boundary spectrum of $\hat{h}_{x}\left(k_{z}\right)$ as a function of $k_{z}$. Bulk (boundary) states are shown in gray (purple). In (c) and (d), we used $t_{x}=t, t_{x z} / t_{x}=0.45$ [cf. Eq. (5)] and $t_{z} / t_{x}=0.03$ to open a gap [43]. The inset shows the spectrum for $t_{z} / t_{x}=0$. (e) The area $S$ enclosing the interface between a nontrivial 1D TI and the vacuum (top) and the domain wall configuration in the $k_{z}$-parameter space that describes it (bottom). In addition, the charge density $\rho$ at half-filling is sketched.

$\tilde{\mathbf{k}}=\left(k_{z}, k_{w}\right)$,

$$
\hat{H}^{4 \mathrm{D}}=\sum_{\tilde{\mathbf{m}}, \tilde{\mathbf{k}}}\left[\hat{H}_{x z}(\tilde{\mathbf{m}}, \tilde{\mathbf{k}})+\hat{H}_{y w}(\tilde{\mathbf{m}}, \tilde{\mathbf{k}})+\Delta \hat{H}_{x y}^{b}(\tilde{\mathbf{m}}, \tilde{\mathbf{k}})\right]
$$

where $\tilde{\mathbf{m}}=\left(m_{x}, m_{y}\right)$,

$$
\begin{aligned}
\hat{H}_{\mu \nu}(\tilde{\mathbf{m}}, \tilde{\mathbf{k}})= & J_{\mu \nu}^{-} c_{\tilde{\mathbf{m}}+\mathbf{e}_{\mu}, \tilde{\mathbf{k}}}^{\dagger} c_{\tilde{\mathbf{m}}, \tilde{\mathbf{k}}}+J_{\mu \nu}^{+} c_{\tilde{\mathbf{m}}-\mathbf{e}_{\mu}, \tilde{\mathbf{k}}}^{\dagger} c_{\tilde{\mathbf{m}}, \tilde{\mathbf{k}}}, \\
\Delta \hat{H}_{x y}^{b}(\tilde{\mathbf{m}}, \tilde{\mathbf{k}})= & \left(e^{i m_{x} b}-1\right) J_{y w}^{-} c_{\tilde{\mathbf{m}}+\mathbf{e}_{y}, \tilde{\mathbf{k}}}^{\dagger} c_{\tilde{\mathbf{m}}, \tilde{\mathbf{k}}} \\
& +\left(e^{-i m_{x} b}-1\right) J_{y w}^{+} c_{\tilde{\mathbf{m}}-\mathbf{e}_{y}, \tilde{\mathbf{k}}}^{\dagger} c_{\tilde{\mathbf{m}}, \tilde{\mathbf{k}}}
\end{aligned}
$$

and $J_{\mu \nu}^{ \pm}=t_{\mu} \pm(-1)^{m_{\mu}} 2 t_{\mu \nu} \cos \left(k_{\nu}\right)$.

We first illustrate the $(2 \mathrm{D} \rightarrow 1 \mathrm{D})$ reduction of the $2 \mathrm{D}$ Creutz model $\hat{H}_{\mu \nu}(\tilde{\mathbf{m}}, \tilde{\mathbf{k}})$ to the $1 \mathrm{D}$ Hamiltonian $\hat{h}_{\mu}\left(k_{v}\right)=$ $\sum_{m_{\mu}} \hat{H}_{\mu \nu}(\tilde{\mathbf{m}}, \tilde{\mathbf{k}})$, where $k_{v}$ is treated as an external parameter [46]. The descendant Hamiltonian $\hat{h}_{\mu}\left(k_{v}\right)$ defines a 1D chain with a unit cell with two degrees of freedom, identical to the Su-Schrieffer-Heeger (SSH) chain [47]. The topological index pertaining to the 1D SSH model is the bulk dipole moment $P_{\mu}\left(k_{v}\right)$ (also known as polarization), which can be calculated using the Wilson loop formalism [32,48]. As a function of $k_{v}$, the bulk dipole $P_{\mu}\left(k_{v}\right) \bmod 1$ takes two values of 0 and $1 / 2$ with the quantization imposed by chiral symmetry; this ensures the existence of gap closing points in the $\left(k_{\mu}, k_{v}\right)$-parameter space, i.e., at the 2D Dirac cones [cf. Fig. 1(b)]. An on-site potential $(-1)^{m_{\mu}} V_{0} c_{\mathbf{m}}^{\dagger} c_{\mathbf{m}}$ (or similarly a nonzero hopping $t_{v}$ [43] in the Creutz model) breaks chiral symmetry and gaps the spectrum. As a consequence, the 
$\left(k_{\mu}, k_{v}\right)$-parameter space acquires a well-defined first Chern number $c_{1}=\int_{0}^{2 \pi} d k_{v} \partial_{k_{v}} P_{\mu}\left(k_{v}\right)$, given by the integral of the change in dipole over the entire descendant 1D family $\hat{h}_{\mu}\left(k_{v}\right)$, see Fig. 1(c) [21,24,49]. Subsequently, the adiabatic evolution of $\hat{h}_{\mu}\left[k_{v}(t)\right]$ along $k_{v}$ dynamically realizes the 2D quantum Hall effect, dubbed topological pumping [10,21,24], see Table I.

A nonvanishing bulk dipole $P_{\mu}\left(k_{v}\right) \neq 0$ has corresponding boundary effects where 0D subgap states appear at the material's boundary, see Fig. 1(d) [49]. Using the formula of Jackiw-Rebbi [42], we calculate the charge $q_{S}$ accumulated in a region $S$ enclosing the boundary [see Fig. 1(e)] by linearizing the dynamics around zero energy to obtain the low-energy Hamiltonian $\hat{h}\left(k_{\nu}\right)=\sum_{\left|k_{\mu}\right| \leqslant \Lambda} \mathbf{d} \cdot \boldsymbol{\sigma}$ where $\mathbf{d}=$ $\left\{v\left(k_{v}\right) k_{\mu}, \mu_{1}\left(k_{v}\right), \mu_{0}\left(k_{v}\right)\right\}$ is a real-valued vector [50], $\sigma=$ $\left\{\sigma_{y}, \sigma_{x}, \sigma_{z}\right\}$ are three anticommuting matrices $\left\{\sigma_{i}, \sigma_{j}\right\}=2 \delta_{i j}$, and $\Lambda$ is a cutoff energy scale. The accumulated charge $q_{S}$ is

$$
q_{S}=\frac{1}{2 \pi} \int_{\tilde{S}} \hat{\mathbf{d}} \cdot\left(\partial_{k_{\mu}} \hat{\mathbf{d}} \times \partial_{r_{\mu}} \hat{\mathbf{d}}\right) d k_{\mu} d r_{\mu},
$$

where $\hat{\mathbf{d}}=\frac{\mathbf{d}}{|\mathbf{d}|}$ and $\tilde{S}=S[-\Lambda, \Lambda]$ is the integration domain. The interface between two insulators in real space is equivalent to a domain wall in the $k_{v}$-parameter space [49], see Fig. 1(e). Thus, Eq. (7) is the first Chern (Berry) flux attached to the region defined by $\tilde{S}$ in the $2 \mathrm{D}$ Brillouin zone (BZ) of the ancestor model. Importantly, $\tilde{S}$ does not cover the entire 2D BZ of the ancestor model. Hence, in the limit where chiral symmetry is restored, $\left|q_{S}\right|$ becomes quantized to two values of $1 / 2$ or 0 , that correspond to encircling or not encircling a singularity with $\pm 1 / 2$ Berry flux. As a consequence, the 1D family $\hat{h}_{\mu}\left(k_{v}\right)$ is divided into a trivial and a nontrivial region with $q_{S}=0$ or $1 / 2$, respectively, in accord with the value of the bulk dipole $q_{S}=\int_{S} \partial_{r_{\mu}} P_{\mu} d r_{\mu}$, cf. Fig. 1(c). This is known as the bulk-boundary correspondence of $1 \mathrm{D} \mathbb{Z}$ TIs, i.e., the relation between the quantized bulk dipole and the charge at the boundary [50]. Note that Eq. (7) also describes the accumulation of nontopological charge at the boundary between two insulators (Tamm states), arising from surface polarizability [51].

In similitude to the $(2 \mathrm{D} \rightarrow 1 \mathrm{D})$ reduction above, the main goal of this Rapid Communication is to demonstrate that the 4D Hamiltonian (1) leads to 2D second-order TIs. A $(4 \mathrm{D} \rightarrow 2 \mathrm{D})$ reduction of Eq. (1) yields the $2 \mathrm{D}$ descendant family $\hat{h}_{x y}^{b}(\tilde{\mathbf{k}})=\sum_{\tilde{\mathbf{m}}}\left[\hat{H}_{x z}(\tilde{\mathbf{m}}, \tilde{\mathbf{k}})+\hat{H}_{y w}(\tilde{\mathbf{m}}, \tilde{\mathbf{k}})+\right.$ $\left.\Delta \hat{H}_{x y}^{b}(\tilde{\mathbf{m}}, \tilde{\mathbf{k}})\right]$, describing a square lattice on the $x y$ plane made out of SSH chains [cf. Eq. (5)] in both the $x$ and the $y$ directions and where each $x y$ plaquette is threaded by a magnetic flux $b$, see Fig. 2(a). Assuming $b=\pi$, the topological invariant of the resulting 2D descendant $\hat{h}_{x y}^{\pi}(\tilde{\mathbf{k}})$ is associated with a quantized bulk quadrupole moment $Q_{x y}$ (either 0 or $\pm \frac{1}{2}$ ), which can be calculated using nested Wilson loops [32,38,52], see Fig. 2(b). Breaking chiral symmetry with an on-site potential $\hat{V}(\mathbf{m})$ [similarly with hopping $t_{z} \neq$ $0 \neq t_{w}$ in Eq. (1)] results in a $4 \mathrm{D} \mathrm{CI}$ with a well-defined second Chern number [53] $c_{2}=\int_{\mathbb{T}^{2}} \partial_{k_{z}} \partial_{k_{w}} Q_{x y}(\tilde{\mathbf{k}}) d^{2} \tilde{\mathbf{k}}$, see Fig. 2(b). Hence, an adiabatic evolution of $\hat{h}_{x y}^{\pi}(\tilde{\mathbf{k}})$ over a closed surface in the $\tilde{\mathbf{k}}$-parameter space dynamically realizes
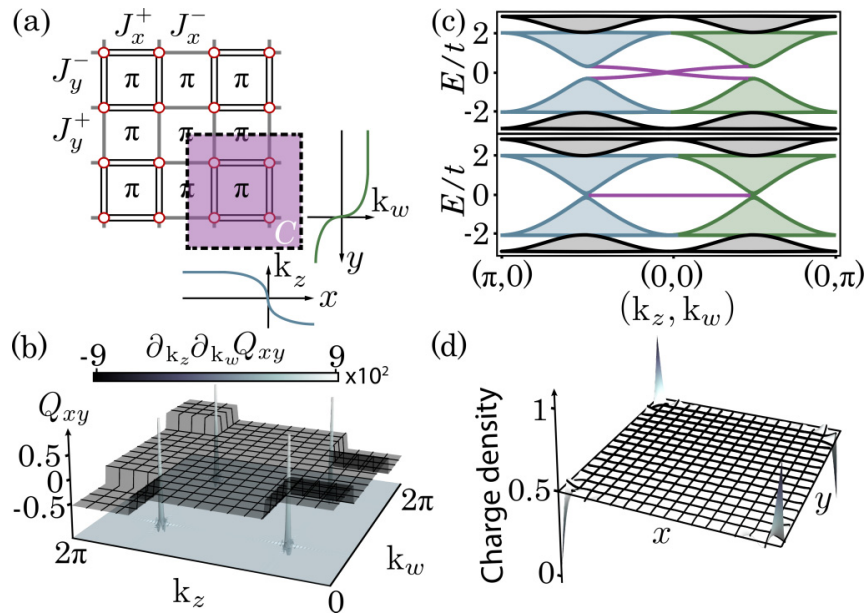

(d)

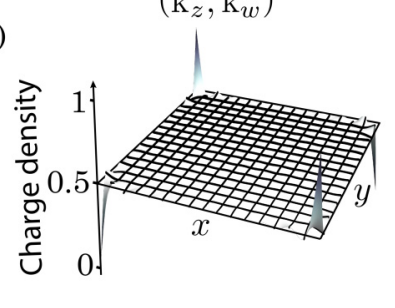

FIG. 2. The descendant 2D family. (a) The descendant model $\hat{h}_{x y}^{\pi}(\tilde{\mathbf{k}})$. Single (double) lines denote hopping amplitude $J_{\mu}^{-}\left(J_{\mu}^{+}\right)$. The area $C$ enclosing the interface of the 2D material with the vacuum (purple square) as well as the corresponding domain-wall configuration in $\tilde{\mathbf{k}}$ are shown. (b) The quadrupole moment $Q_{x y}(\tilde{\mathbf{k}})$ of $\hat{h}_{x y}^{\pi}(\tilde{\mathbf{k}})$ and the curvature $\partial_{k_{z}} \partial_{k_{w}} Q_{x y}(\tilde{\mathbf{k}})$ associated with the second Chern number $c_{2}$; the total area under the curvature is an integer. (c) The open boundary spectrum of $\hat{h}_{x y}^{\pi}(\tilde{\mathbf{k}})$ for a selected path in $\tilde{\mathbf{k}}$. Depicted are bulk bands (gray), upper/lower edge states (blue), right/left edge states (green), and corner states (purple). The top (bottom) spectrum has broken (preserved) chiral symmetry with $t_{z}=t_{w}=0.03 t\left(t_{z}=t_{w}=0\right)$. (d) The charge density of $\hat{h}_{x y}^{\pi}(\tilde{\mathbf{k}})$ at half-filling and $\tilde{\mathbf{k}}=(0,0)$ has $\pm 1 / 2$ charge deviation at the corners. In (b)-(d), we used $t_{x}=t, t_{x} / t_{y}=1$, and $t_{x z} / t_{x}=t_{y w} / t_{y}=0.45$. In (b), we used $t_{z}=t_{w}=0.001 t$ to minimally open the gap.

the 4D quantum Hall effect, dubbed 2D topological pumping $[18,25,26]$, see Table I.

The bulk responses of the descendant 2D pump family have associated boundary phenomena [26] where: (i) 1D edge states, i.e., states of co-dimension $h=1$, appear in the spectrum, and (ii) OD corner states, i.e., states of co-dimension $h=2$, disperse as a function of $\tilde{\mathbf{k}}$, see Figs. 2(c) and 2(d). We generalize Eq. (7) and derive [53] the charge accumulation $q_{C}$ in a region $C$ enclosing the corner of the $2 \mathrm{D}$ material [cf. Fig. 2(a)] with low-energy Hamiltonian $\hat{h}(\tilde{\mathbf{k}})=\int_{|\mathbf{k}| \leqslant \Lambda} \mathbf{d}$. $\boldsymbol{\Gamma} d^{2} k$, where $\mathbf{d}=\left\{\mu_{0}(\tilde{\mathbf{k}}), \mu_{1}(\tilde{\mathbf{k}}), \mu_{2}(\tilde{\mathbf{k}}), v_{x}(\tilde{\mathbf{k}}) k_{x}, v_{y}(\tilde{\mathbf{k}}) k_{y}\right\}$ is a real-valued vector [53], $\boldsymbol{\Gamma}=\left\{\Gamma_{0}, \ldots, \Gamma_{4}\right\}$ are five anticommuting matrices $\left\{\Gamma_{\mu}, \Gamma_{\nu}\right\}=2 \delta_{\mu \nu}$, and $\Lambda$ is a cutoff energy scale. We obtain

$$
q_{C}=\int_{\tilde{C}} \hat{\mathbf{d}} \cdot\left(\partial_{k_{x}} \hat{\mathbf{d}} \times \partial_{k_{y}} \hat{\mathbf{d}} \times \partial_{r_{x}} \hat{\mathbf{d}} \times \partial_{r_{y}} \hat{\mathbf{d}}\right) d^{2} k d^{2} r,
$$

where $\hat{\mathbf{d}}=\frac{\mathbf{d}}{|\mathbf{d}|}$ and $\tilde{C}=C \times[-\Lambda, \Lambda]^{2}$ is the integration domain. Since the corner of the material can be expressed as the intersection of two edges, i.e., two domain walls in the $\tilde{\mathbf{k}}$ parameter space [cf. Fig. 2(a)], $q_{C}$ is equivalent to the second Chern flux attached to the region defined by $\tilde{C}$ in the $4 \mathrm{D}$ $\mathrm{BZ}$ of the ancestor Hamiltonian. Note that $\tilde{C}$ does not cover the entire 4D BZ of the ancestor CI. Hence, in the limit where chiral symmetry is restored, $\left|q_{C}\right|$ becomes quantized to $1 / 2$ or 0 , corresponding to encircling or not encircling a 4D singularity with $\pm \frac{1}{2}$ second Chern flux. For the $2 \mathrm{D}$ 
descendant $\hat{h}_{x y}^{\pi}(\tilde{\mathbf{k}})$, we find that the $\tilde{\mathbf{k}}$-parameter space is divided into trivial regions with $\left|q_{C}\right|=0$ and nontrivial regions with $\left|q_{C}\right|=1 / 2$; the latter exhibiting zero-energy states localized at the corners, in accord with the value of the bulk quadrupole $q_{C}=\int_{C} \partial_{r_{x}} \partial_{r_{y}} Q_{x y} d^{2} \mathbf{r}$, cf. Figs. 2(b)-2(d).

The connection between charge accumulation at the corner and the second Chern flux is a key outcome of this Rapid Communication. In general, such charges can arise due to bulk properties as well as due to boundary effects. The charge accumulation $q_{C}$ in a finite macroscopic 2D material interfaced with another material, e.g, the vacuum, can be calculated using the electric multipole moments [32],

$$
q_{C}=\int_{C}\left(\rho_{\text {bulk }}+\rho_{\partial}+\rho_{\partial \partial}\right) d^{2} \mathbf{r},
$$

where $\rho_{\text {bulk }}=-\nabla \cdot \vec{P}+\frac{1}{2} \partial_{r_{\mu}} \partial_{r_{\nu}} Q_{\mu \nu}$ are the contributions due to the bulk dipole $\vec{P}$ and quadrupole $Q_{x y}$ densities, $\rho_{\partial}=\hat{\vec{n}}$. $\left.\vec{P}\right|_{\partial}-\left.\hat{n}_{\mu} \partial_{r_{\nu}} Q_{\mu \nu}\right|_{\partial}$ are the contributions due to a "free" edge dipole $\left.\hat{\vec{n}} \cdot \vec{P}\right|_{\partial}$ and quadrupole $\left.\hat{n}_{\mu} \partial_{r_{\nu}} Q_{\mu \nu}\right|_{\partial}$ densities, and $\rho_{\partial \partial}=$ $\frac{1}{2} \hat{n}_{\mu}^{\alpha} \hat{n}_{\nu}^{\beta} Q_{\mu \nu}$ is the contribution due to a point charge created by a free quadrupole density at the intersection of two edges with normal vectors $\hat{\vec{n}}^{\alpha}$ and $\hat{\vec{n}}^{\beta}$. Hence, a nontrivial value of $q_{C}$ can originate from a combination of bulk and surface terms [53].

For the 2D Hamiltonian $\hat{h}_{x y}^{\pi}(\tilde{\mathbf{k}})$, the only nonvanishing contributions to the corner charge $q_{C}$ arise from a quantized bulk quadrupole $Q_{x y}$. On the other hand, starting from the 4D ancestor Hamiltonian (1) with $b=0$ we obtain, using $(4 \mathrm{D} \rightarrow 2 \mathrm{D})$ reduction, a $2 \mathrm{D}$ descendant $\hat{h}_{x y}^{0}(\tilde{\mathbf{k}})$ composed of coupled SSH chains along the $x$ and $y$ directions, see Fig. 3(a). This model $\hat{h}_{x y}^{0}(\tilde{\mathbf{k}})$ has zero bulk quadrupole $Q_{x y}$ but nonzero edge dipoles $\left.\vec{P}\right|_{\partial}$ that result in two distinct phases with $q_{C}=0$ or $1 / 2$; the latter having zero-energy states localized at the corners that merge into the bulk or edge spectrum at gap closing points [see Figs. 3(b) and 3(c)]. As a third example, we start from Eq. (1) with $b=0$ and thread a $\pi$ flux through each $x w$ plaquette. This leads, using $(4 \mathrm{D} \rightarrow 2 \mathrm{D})$ reduction, to a $2 \mathrm{D}$ descendant family denoted by $\hat{h}_{x y}^{0, \pi}(\tilde{\mathbf{k}})$, describing SSH chains $\hat{h}_{x}\left(k_{z}\right)$ along the $x$ direction, coupled to alternating SSH chains $\hat{h}_{y}\left(k_{w}+\pi x\right)$ in the $y$ direction, see Fig. 3(d). The charge $q_{C}$ is now a combination of bulk and surface terms that sum to quantized values 0 or $1 / 2$, see Fig. 3(e). The spectrum is separated by bulk- or edge-gap closing points into regions characterized by the appearance of zero-energy states localized at the corners, see Fig. 3(f). In all three cases, the corner charge accumulation is associated with a nontrivial value of the second Chern flux [53].

The demonstrated relationship between the 4D chiral semimetal (1) and the 2D second-order TIs offers a plethora of generalizations. Namely, a wide variety of 4D ancestor models can be constructed where various planes are threaded with $2 \pi / q$ fluxes (where $q$ is an even integer), and different directions are dimensionally reduced. An even $q$ is crucial for obtaining a low-energy theory corresponding to decoupled Dirac cones [20], thus, defining regions in the BZ that are separated by gap closures. Moreover, our results readily extend to $3 \mathrm{D}$, explaining the appearance of hinge modes and relating corner states to a six-dimensional $\mathrm{CI}$ and its third Chern number $[19,30]$. These charges arise from a combination of
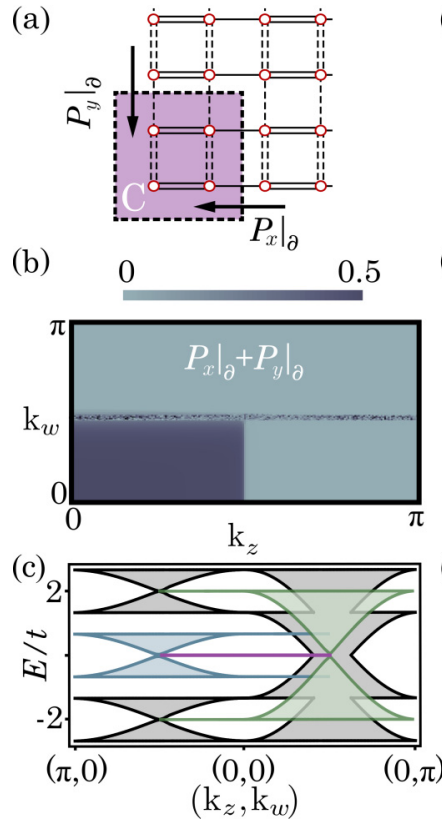

$(0, \pi) \quad(\pi, 0)$ (d)
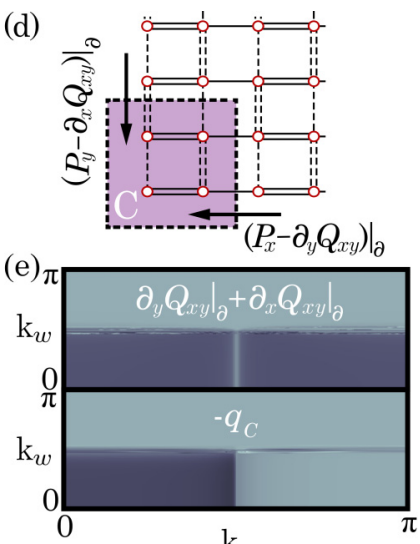

$\mathrm{k}$

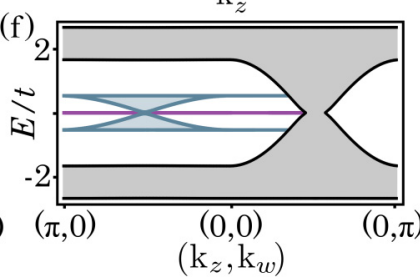

FIG. 3. Descendant 2D families and their multipole description. (a) The model $\hat{h}_{x y}^{0}(\tilde{\mathbf{k}})$. Single (double) lines denote hopping amplitude $J_{\mu}^{-}\left(J_{\mu}^{+}\right)$. Dashed (solid) lines denote $x(y)$ hopping amplitudes. (b) The charge $q_{C}$ as a function of $\tilde{\mathbf{k}}$, showing two distinct regions with $q_{C}=0$ and $q_{C}=1 / 2$. Discontinuities around $\mathrm{k}_{w}=\pi / 2$ are due to bulk bands approaching zero energy [cf. (c)]. (c) The open boundary spectrum of $\hat{h}_{x y}^{0}(\tilde{\mathbf{k}})$ for a selected path in $\tilde{\mathbf{k}}$. Colors denote bulk/boundary states as in Fig. 2(c). (d) The model $\hat{h}_{x y}^{0, \pi}(\tilde{\mathbf{k}})$. Lines denote hopping amplitudes as in (a). (e) Upper: The contribution to $q_{C}$ from the quadrupole moment. Lower: The charge $q_{C}$ as a function of $\tilde{\mathbf{k}}$. (f) The open boundary spectrum of $\hat{h}_{x y}^{0, \pi}(\tilde{\mathbf{k}})$ for a selected path in $\tilde{\mathbf{k}}$, exhibiting regions with: (i) zero-energy states localized at the upper/lower left corner, (ii) zero-energy states localized at the upper/lower right corner, and (iii) no zero-energy solutions. Colors denote bulk/boundary states as in Fig. 2(c). In (b), (c), (e), and (f), we have used $t_{x}=t, t_{y} / t_{x}=1 / 10, t_{x z} / t_{x}=t_{y w} / t_{y}=0.45$, and an on-site staggered mass $V_{0}=0.001 t$.

octapole, quadrupole, and dipole moments. Equivalently, our procedure generates multiple topological pump realizations where charge transport is proportional to the modulation of the bulk dipole, quadrupole, and octapole moments. This naturally explains the appearance of surface, hinge, and corner modes (cf. Fig. 2(b) and Ref. [18]).

In this Rapid Communication, we reveal a connection among the physics of high-order TIs, topological pumps, and Chern insulators using dimensional reduction. This allows us to define a topological index associated with the charge accumulation at the corners, leading to a simple unifying understanding of standard TIs and higher-order TIs. It engenders a single ancestor high-dimensional insulator and uses dimensional reduction as a tool to find new higher-order TIs, each with its own low-dimensional description. Comparing our invariant with the electric multipole expansion, we establish that corner charges arise from the combination of bulk and surface multipole moments. 
We thank H. M. Price, I. Mondragon, M. Soriente, T. Wolf, C. Mudry, and M. Rechtsman for fruitful discussions.
We acknowledge financial support from the Swiss National Science Foundation Grant No. PP00P2_163818.
[1] M. Z. Hasan and C. L. Kane, Rev. Mod. Phys. 82, 3045 (2010).

[2] X.-L. Qi and S.-C. Zhang, Rev. Mod. Phys. 83, 1057 (2011).

[3] T. Ozawa, H. M. Price, A. Amo, N. Goldman, M. Hafezi, L. Lu, M. C. Rechtsman, D. Schuster, J. Simon, O. Zilberberg et al., Rev. Mod. Phys. 91, 015006 (2019).

[4] A. Kitaev, in Advances in Theoretical Physics: Landau Memorial Conference, edited by V. Lebedev and M. Fei'gelman, AIP Conf. Proc. No. 1134 (AIP, New York, 2009), pp. 22-30.

[5] S. Ryu, A. P. Schnyder, A. Furusaki, and A. W. Ludwig, New J. Phys. 12, 065010 (2010).

[6] A. Altland and M. R. Zirnbauer, Phys. Rev. B 55, 1142 (1997).

[7] L. Fu, Phys. Rev. Lett. 106, 106802 (2011).

[8] M. Kremer, I. Petrides, E. Meyer, M. Heinrich, O. Zilberberg, and A. Szameit, Nat. Commun. 11, 907 (2020).

[9] J. Bellissard, D. Herrmann, and M. Zarrouati, Directions in Mathematical Quasicrystals, edited by M. Baake and R. V. Moody, CRM Monograph Series Vol. 13 (AMS, Providence, 2000).

[10] Y. E. Kraus and O. Zilberberg, Phys. Rev. Lett. 109, 116404 (2012).

[11] Y. E. Kraus and O. Zilberberg, Nat. Phys. 12, 624 (2016).

[12] J. Kruthoff, J. de Boer, J. van Wezel, C. L. Kane, and R.-J. Slager, Phys. Rev. X 7, 041069 (2017).

[13] K. Shiozaki, M. Sato, and K. Gomi, Phys. Rev. B 95, 235425 (2017).

[14] A. Alexandradinata, Z. Wang, and B. A. Bernevig, Phys. Rev. X 6, 021008 (2016).

[15] J. Bellissard, From Number Theory to Physics (Springer, Berlin, 1992), pp. 538-630.

[16] E. Prodan, Phys. Rev. B 91, 245104 (2015).

[17] C.-K. Chiu, J. C. Y. Teo, A. P. Schnyder, and S. Ryu, Rev. Mod. Phys. 88, 035005 (2016).

[18] Y. E. Kraus, Z. Ringel, and O. Zilberberg, Phys. Rev. Lett. 111, 226401 (2013).

[19] I. Petrides, H. M. Price, and O. Zilberberg, Phys. Rev. B 98, 125431 (2018).

[20] D. J. Thouless, M. Kohmoto, M. P. Nightingale, and M. den Nijs, Phys. Rev. Lett. 49, 405 (1982).

[21] D. J. Thouless, Phys. Rev. B 27, 6083 (1983).

[22] Y. Kraus, Phys. Rev. Lett. 109, 106402 (2012).

[23] M. Verbin, O. Zilberberg, Y. Lahini, Y. E. Kraus, and Y. Silberberg, Phys. Rev. B 91, 064201 (2015).

[24] M. Lohse, C. Schweizer, O. Zilberberg, M. Aidelsburger, and I. Bloch, Nat. Phys. 12, 350 (2016).

[25] M. Lohse, C. Schweizer, H. M. Price, O. Zilberberg, and I. Bloch, Nature (London) 553, 55 (2018).

[26] O. Zilberberg, S. Huang, J. Guglielmon, M. Wang, K. P. Chen, Y. E. Kraus, and M. C. Rechtsman, Nature (London) 553, 59 (2018).

[27] X.-L. Qi, T. L. Hughes, and S.-C. Zhang, Phys. Rev. B 78, 195424 (2008).

[28] S. Ryu, C. Mudry, C.-Y. Hou, and C. Chamon, Phys. Rev. B 80, 205319 (2009).
[29] M. Lin and T. L. Hughes, Phys. Rev. B 98, 241103(R) (2018).

[30] K. Hashimoto, X. Wu, and T. Kimura, Phys. Rev. B 95, 165443 (2017).

[31] J. Langbehn, Y. Peng, L. Trifunovic, F. von Oppen, and P. W. Brouwer, Phys. Rev. Lett. 119, 246401 (2017).

[32] W. A. Benalcazar, B. A. Bernevig, and T. L. Hughes, Science 357, 61 (2017).

[33] L. Trifunovic and P. W. Brouwer, Phys. Rev. X 9, 011012 (2019).

[34] M. Geier, L. Trifunovic, M. Hoskam, and P. W. Brouwer, Phys. Rev. B 97, 205135 (2018)

[35] F. Schindler, Z. Wang, M. G. Vergniory, A. M. Cook, A. Murani, S. Sengupta, A. Y. Kasumov, R. Deblock, S. Jeon, I. Drozdov et al., Nat. Phys. 14, 918 (2018).

[36] Z. Wang, B. J. Wieder, J. Li, B. Yan, and B. A. Bernevig, Phys. Rev. Lett. 123, 186401 (2019).

[37] M. Ezawa, Phys. Rev. B 98, 045125 (2018).

[38] M. Serra-Garcia, V. Peri, R. Süsstrunk, O. R. Bilal, T. Larsen, L. G. Villanueva, and S. D. Huber, Nature (London) 555, 342 (2018).

[39] S. Imhof, C. Berger, F. Bayer, J. Brehm, L. W. Molenkamp, T. Kiessling, F. Schindler, C. H. Lee, M. Greiter, T. Neupert et al., Nat. Phys. 14, 925 (2018).

[40] S. A. A. Ghorashi, X. Hu, T. L. Hughes, and E. Rossi, Phys. Rev. B 100, 020509 (2019).

[41] D. Călugăru, V. Juričić, and B. Roy, Phys. Rev. B 99, 041301(R) (2019).

[42] R. Jackiw and C. Rebbi, Phys. Rev. D 13, 3398 (1976).

[43] Y. Hatsugai and M. Kohmoto, Phys. Rev. B 42, 8282 (1990).

[44] M. Creutz, Phys. Rev. Lett. 83, 2636 (1999).

[45] R. Peierls, Z. Phys. 80, 763 (1933).

[46] We drop the dependence on the remaining coordinates $m_{\sigma}$ and $k_{\rho}$ since the Hamiltonian $\hat{h}_{\mu}\left(k_{v}\right)$ is independent of them.

[47] W. P. Su, J. R. Schrieffer, and A. J. Heeger, Phys. Rev. Lett. 42, 1698 (1979).

[48] R. D. King-Smith and D. Vanderbilt, Phys. Rev. B 47, 1651(R) (1993).

[49] D. Vanderbilt and R. D. King-Smith, Phys. Rev. B 48, 4442 (1993).

[50] See Supplemental Material at http://link.aps.org/supplemental/ 10.1103/PhysRevResearch.2.022049 where we derive the charge accumulation at the corner of $2 \mathrm{D}$ materials, show the multipole description of macroscopic material, and calculate the second Chern forms of the three examples considered in the main text.

[51] G. M. Graf and J. Shapiro, Commun. Math. Phys. 363, 829 (2018).

[52] Taking $t_{z}>0$ and $t_{x z}=0$ in the $2 \mathrm{D}$ ancestor Hamiltonian $\hat{H}_{\mu \nu}(\tilde{\mathbf{m}}, \tilde{\mathbf{k}})$ leads, upon dimensional reduction, to a topologically trivial model. However, localized states appear at its interface with an insulator with $t_{z}<0$ and $t_{x z}=0$ due to a combination nonzero surface and bulk dipole moments.

[53] W. A. Benalcazar, B. A. Bernevig, and T. L. Hughes, Phys. Rev. B 96, 245115 (2017). 